\title{
Hot DQ white dwarfs: a pulsational test of the mixing scenario for their formation
}

\author{
A. D. Romero ${ }^{1,2}$, A. H. Córsico ${ }^{1,2}$, L. G. Althaus ${ }^{1,2}$ \& E. \\ García-Berro $^{3,4}$ \\ ${ }^{1}$ Facultad de Ciencias Astronómicas y Geofísicas, Universidad Nacional de La Plata, Argentina \\ ${ }^{2}$ Instituto de Astrofísica La Plata, IALP, CONICET-UNLP \\ ${ }^{3}$ Departament de Fisica Aplicada, Escola Politécnica Superior de Castelldefels, Universitat \\ Politécnica de Catalunya \\ ${ }^{4}$ Institut d'Estudis Espacials de Catalunya \\ email: aromero@fcaglp.unlp.edu.ar
}

Hot DQ white dwarfs constitute a new class of white dwarf stars, uncovered recently within the framework of SDSS project. There exist nine of them, out of a total of several thousands white dwarfs spectroscopically identified. Recently, three hot DQ white dwarfs have been reported to exhibit photometric variability with periods compatible with pulsation $g$-modes. In this contribution, we presented the results of a non-adiabatic pulsation analysis of the recently discovered carbon-rich hot DQ white dwarf stars. Our study relies on the full evolutionary models of hot DQ white dwarfs recently developed by Althaus et al. (2009), that consistently cover the whole evolution from the born-again stage to the white dwarf cooling track. Specifically, we performed a stability analysis on white dwarf models from stages before the blue edge of the DBV instability strip $\left(T_{\text {eff }} \approx 30000 \mathrm{~K}\right)$ until the domain of the hot DQ white dwarfs $(18000-24000 \mathrm{~K})$, including the transition DB $\rightarrow$ hot DQ white dwarf. We explore evolutionary models with $M_{*}=0.585 M_{\odot}$ and $M_{*}=0.87 M_{\odot}$, and two values of thickness of the He-rich envelope $\left(M_{\mathrm{He}}=2 \times 10^{-7} M_{*}\right.$ and $\left.M_{\mathrm{He}}=10^{-8} M_{*}\right)$.

We found that at epochs in which the models have He-dominated atmospheres, they exhibit $g$ mode pulsations typical of DBV stars. When the white dwarf models become carbon-dominated atmospheres, they continue being pulsationally unstable with similar characteristics than DB models, showing overstable $g$-modes primarily driven through the $\kappa$-mechanism due to the partial ionization of $\mathrm{C}$. The blue edge of DQVs is hotter for less massive models and for thinner $\mathrm{He}$ envelopes. In particular, the instability domains of DBVs and DQVs are clearly separated in the $T_{\text {eff }}-\Pi$ plane for the case of models with $M_{\mathrm{He}}=10^{-8} M_{*}$, but the transition is continuous in the case in which $M_{\mathrm{He}}=2 \times 10^{-7} M_{*}$. The periods detected in SDSS J142625.70+575218.4 and SDSS J220029.08-074121.5 are well within our theoretical ranges of excited periods, but the period at $\Pi \sim 1052 \mathrm{~s}$ corresponding to SDSS J234843.300-94245.3 is long in excess, and is not well accounted for by our models. Finally, our work demonstrate that the diffusive/convective mixing scenario not only is able to nicely explain the origin of hot DQ white dwarfs, but it also accounts for the variability of these stars. We caution that the results of the present work could somewhat change if the frozen convection approximation were relaxed in our stability analysis. This calculations provide strong support to the convective-mixing picture for the formation of hot DQs. We found theoretical evidence that a fraction of pulsating DB white dwarfs - those characterized by thin He-rich envelopes - would become pulsating DQ white dwarfs.

\section{References}

Althaus, L. G., Garcia-Berro, E., Córsico, et al., 2009, ApJL, 693, L23 